\title{
Gobernanza en espacios periurbanos a través de la iniciativa europea LEADER. El caso de la Comarca madrileña de Las Vegas.
}

\author{
Luis Daniel ABAD ARAGÓN \\ Departamento de Geografía Humana \\ Universidad Complutense de Madrid \\ ldaniel_aragon@yahoo.es
}

Recibido: 28 de septiembre de 2013

Enviado a evaluar: 8 de enero de 2014

Aceptado: 30 de abril de 2014

\section{RESUMEN}

Los procesos de expansión experimentados por las ciudades durante las últimas décadas han generado una nueva realidad urbano-regional, en la cual se insertan los espacios periurbanos. Considerados inicialmente una tercera corona de límites imprecisos hacia la que se diluía la difusión metropolitana, son contemplados actualmente como una nueva categoría espacial, como un tercer espacio que actúa de interfaz entre lo urbano y lo rural, y que se caracteriza por su complejidad funcional. El objeto de este artículo es analizar nuevas estructuras de gobernanza para el desarrollo de espacios periurbanos mediante el estudio de los Grupos de Acción Local, entidades experimentales de gobernanza vinculadas a la Iniciativa Comunitaria LEADER. En concreto, analizamos el papel jugado por el Grupo de Acción Local ARACOVE en la comarca periurbana madrileña de Las Vegas, a través de la realización de 32 entrevistas, así como de técnicas cuantitativas habituales en estudios sobre desarrollo urbano.

Palabras clave: Gobernanza, espacios periurbanos, enfoque Leader, desarrollo territorial

\section{Governance in peri-urban areas through the Community Initiative LEADER. The case of the region of Las Vegas in Madrid}

\begin{abstract}
The processes of expansion experienced by the cities during the last few decades have created a new urban-regional reality, in which they are inserted peri-urban areas. Initially considered a third-ring suburb of indistinct limits toward that was diluted the suburban sprawl, peri-urban areas are referred to now as a new space category, as a tiers espace that serves as an interface between urban and rural, and that is characterized by its functional complexity. The aim of this article is to analyze new governance structures for peri-urban areas development, through the study of the Local Action Groups, experimental governance entities linked to the Community Initiative LEADER. In particular, we analyze the role played by the Local Action Group ARACOVE in peri-urban region of Las Vegas, in Madrid, through the realization of 32 interviews, as well as quantitative techniques common in studies of urban development.
\end{abstract}

Key words: Governance, peri-urban areas, Leader approach, territorial development 


\section{Gouvernance dans espaces périurbaines par l'intermédiaire de l'Initiative Européenne LEADER. Le cas du pays madrilène de Las Vegas}

\section{RÉSUMÉ}

Les processus d'expansion expérimentés par les villes au cours des dernières décennies ont suscité une nouvelle réalité urbain-régional, dans laquelle sont insérés les espaces périurbains. Considérés initialement une troisième couronne de limites imprécis vers laquelle la diffusion métropolitaine s'éloigna, les espaces périurbains sont visés actuellement comme une nouvelle catégorie spatiale, comme un tiers espace qui agit d'interface entre les zones urbaines et les zones rurales, et qui se caractérise par leur complexité fonctionnelle. Le but de cet article est d'analyser nouvelles structures de gouvernance pour le développement d'espaces périurbains grâce à l'étude des Groupes d'Action Locale, des entités expérimentales de gouvernance liées à l'Initiative Communautaire LEADER. En particulier, nous avons analysé le rôle joué par le Groupe d'Action Locale ARACOVE dans le pays périurbain de Las Vegas, à Madrid, par l'intermédiaire de la réalisation de 32 entretiens, ainsi que de techniques quantitatives habituelles dans les études sur le développement urbain.

Mots clés: Gouvernance, espaces périurbaines, approche Leader, développement territoriale

\section{INTRODUCCIÓN}

A lo largo de las últimas décadas, una parte importante de las investigaciones llevadas a cabo en las ciencias sociales se han centrado en la búsqueda de factores que expliquen y den soluciones al fenómeno del desigual desarrollo de los territorios. El presente artículo se enmarca dentro de este campo de estudio mediante el análisis del factor: gobierno del territorio.

Para ello, se recurre al estudio de nuevas formas de gobernar el territorio basadas en la noción de gobernanza, definida como un modelo alternativo de gestionar los asuntos públicos que implica la transformación y la recomposición de la acción pública, mediante la incorporación de nuevos actores de naturaleza no política y de un nuevo sistema de relaciones entre éstos y la Administración en sus distintos niveles de decisión (Le Galés, 1995; Farinós, 2008; Jacquier, 2008).

El artículo, que emana de la Tesis Doctoral realizada por el autor (Abad, 2013), aborda esta cuestión mediante el análisis de los denominados Grupos de Acción Local (GAL), estructuras experimentales de gobernanza e innovación socio-institucional que, creadas a comienzos de los años noventa en el marco de la iniciativa europea LEADER (Liaison Entre Actions de Développement de l'Économie Rurale, en adelante Leader), se basan en la cooperación entre múltiples y diversos actores locales, con el objetivo de lograr un desarrollo equilibrado y sostenible en sus territorios de intervención. En este sentido, se analiza el papel asumido por el GAL AranjuezComarca de Las Vegas (en adelante, Aracove), que se localiza en el periurbano de la región administrativa y urbana de Madrid.

La recogida y tratamiento de información se ha basado en la aplicación de una metodología que aúna técnicas cuantitativas y cualitativas, si bien han sido éstas últimas las que sustentan el grueso de la investigación, dadas las características del objeto de 
estudio. En concreto, se ha empleado como principal herramienta cualitativa la entrevista semiestructurada de tipo abierta y enfocada, que ha sido realizada a un total de 30 agentes locales y regionales de distinta naturaleza, pertenecientes y no pertenecientes a Aracove. Esta técnica ha permitido detectar los diferentes discursos existentes en el territorio sobre tres temas considerados centrales: la percepción sobre el proceso de periurbanización de la comarca; detección de redes formales e informales de actores, organismos e instituciones; y conocimiento y valoración del papel asumido por Aracove en la gobernanza y el desarrollo de la comarca.

\section{EL ENFOQUE LEADER $Y$ SU POTENCIALIDAD PARA LA GOBERNANZA DE ESPACIOS PERIURBANOS}

A partir de la recuperación del territorio como factor de desarrollo, desde finales de los años setenta del siglo pasado evolucionan un conjunto de teorías que, agrupadas alrededor de la denominación de Modelos de Innovación Territorial -MIT(Distritos Industriales; Milieux Innovateurs, Learning Regions...), supondrán la materialización de una serie de propuestas teóricas destinadas a analizar y proponer alternativas de desarrollo para zonas urbano- industriales en declive.

Sin embargo, "a pesar de su apego a las dinámicas institucionales, los MIT se mantienen fieles a una ontología económica basada en las leyes del mercado y en una visión del desarrollo puramente tecnológica" (Moulaert y Nussbaumer, 2005:101), por lo que asimilan el concepto de desarrollo al de crecimiento económico (Boisier, 2004). A lo largo de la última década del siglo XX se propondrá un cambio en este paradigma hacia modelos más holísticos que se agruparán alrededor de un nuevo enfoque: el Desarrollo Territorial Integrado (DTI).

Mediante la incorporación de la idea de desarrollo comunitario, este nuevo enfoque pretende no solo superar las propuestas realizadas por los MIT en el seno del paradigma del Desarrollo Territorial, sino también construir una alternativa a la organización social propuesta por las corrientes teóricas neoliberales basadas en el individualismo.

De esta manera, bajo la perspectiva del DTI se considera que la construcción de políticas púbicas de desarrollo requiere la activación del capital social e institucional presente en los territorios locales, para lo cual se torna necesario habilitar mecanismos innovadores de gobierno que estimulen, integren y regulen la participación de los distintos agentes implicados independientemente de su naturaleza. La gobernanza se convierte así en un proceso necesario para conseguir que las redes socioinstitucionales creadas sean capaces de movilizar al resto de los componentes del capital territorial (capital humano o intelectual; capital empresarial o productivo; capital natural o ecológico), interrelacionándolos y creando sinergias positivas entre ellos, con el objetivo de alcanzar respuestas satisfactorias a sus necesidades de bienestar, de calidad de vida y de justicia social. 


\subsection{MODELOS DE GESTIÓN DE LA GOBERNANZA. EVOLUCIÓN Y CARACTERÍSTICAS GENERALES}

Equiparado tradicionalmente a la noción de gobierno, desde finales de los años setenta el concepto de gobernanza comenzará a diferenciarse de aquella como consecuencia de la crisis del Estado nacional y de su estructura organizativa de tipo burocrático (figura 1).

Figura 1. Evolución de los modelos de gestión de la gobernanza

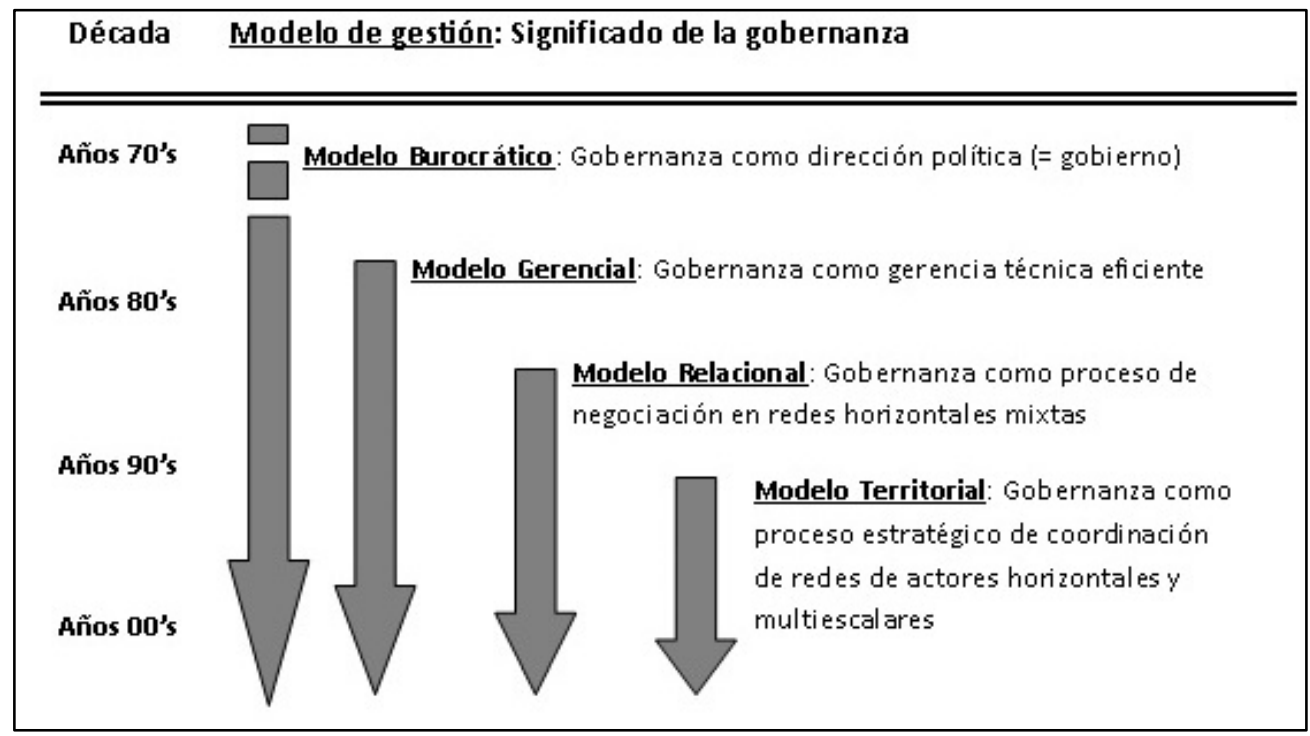

Fuente: Elaboración propia, a partir de Torres y Ramos, 2008

Dicha crisis va a suponer un doble proceso que, por un lado, llevará a la recomposición del propio modelo burocrático previo, que a lo largo de las décadas siguientes irá flexibilizándose y desconcentrando y distribuyendo el poder que hasta entonces había acumulado el Estado nacional, hacia entidades de carácter supranacional -Unión Europea- y hacia entidades de carácter subnacional comunidades autónomas y administración local en el caso español-; por otro lado, la crisis del Estado nacional va a suponer también la apertura de los distintos niveles de la administración pública a la cooperación con agentes no políticos en el proceso de gestión de las políticas públicas. Este último proceso estará asociado a la aparición de modelos alternativos de gobierno vinculados con la gobernanza.

Dichos modelos alternativos estarán influenciados inicialmente por las teorías económicas y políticas conservadoras de corte neoliberal, bajo las cuales se considera que una buena gobernanza debe apoyarse en la reducción del papel del Estado en la administración de los asuntos públicos y su sustitución por agentes del mercado y del 
tercer sector, quienes aplicando estrategias empresariales lograrán una gestión técnica y económica más eficiente de la res pública. Se da forma así a la denominada gobernanza gerencial o empresarial.

En la primera mitad de los años ochenta surgirá desde la economía y la sociología neoinstucionalistas un nuevo modelo de gestión de la gobernanza, que se va a apoyar en el descubrimiento de la red como forma alternativa de administrar los asuntos públicos; alternativa al Estado del modelo de gobierno burocrático y alternativa al mercado propuesto por las teorías gerencialistas. Este enfoque neoinstitucional propone la construcción de espacios relacionales abiertos a la participación de actores públicos, privados y civiles, dentro de los cuales se llevan a cabo procesos horizontales de negociación y de cooperación. Surge así la gobernanza relacional.

Finalmente, a partir de los años noventa, y vinculado a los procesos de globalización y de construcción europea, surgirá un tercer modelo de gestión de la gobernanza que supone fundamentalmente la incorporación de un enfoque estratégico-territorial a los modelos relacionales. Estas nuevas propuestas también consideran oportuno generar redes mixtas de cooperación horizontal, pero inciden en la necesidad de que dichas redes, que operan desde las distintas escalas de decisión política -local, regional, nacional o supranacional- estén interconectadas. De esa manera, desde el enfoque estratégico-territorial se sostiene que la gobernanza es ante todo un proceso estratégico de coordinación de redes de actores que operan de manera horizontal y multiescalar. Aparece así la gobernanza territorial.

\subsection{LA INICIATIVA EUROPEA LEADER COMO INSTRUMENTO EXPERIMENTAL PARA LA GOBERNANZA EN ESPACIOS PERIURBANOS}

Tanto el enfoque institucionalista como el estratégico-territorial serán incorporados en los programas e iniciativas experimentales con los que la Unión Europea viene ensayando durante las últimas décadas del siglo XX sobre diferentes tipos de territorios en crisis (rurales, de expansión periurbana, urbanos, espacios transfronterizos, etc.), entre los que destaca la iniciativa Leader a partir del año 1991.

Esta iniciativa ha evolucionado como programa experimental de desarrollo rural integrado entre 1991 y 2006 a lo largo de tres fases: Leader I (1991-1993); Leader II (1994-1999) y Leader + (2000-2006). En el actual periodo de programación (20072013), sin embargo, la Comisión Europea decide convertirlo en un eje metodológico experimental para la aplicación de la actual Política de Desarrollo Rural europea, una política cuya implementación no se centra sólo sobre los espacios rurales, sino que desde las instancias europeas se promueve con creciente interés su aplicación sobre los espacios de expansión periurbana (Comisión Europea, 2010; González de Canales, 2011; Artman et al., 2012; ENRD, 2013). 
Los tres elementos principales que definen a Leader son:

- La delimitación de un territorio de actuación (comarca Leader);

- el establecimiento de una estructura partenarial de gobernanza territorial (Grupos de Acción Local);

- y la construcción conjunta de una estrategia de desarrollo (Estrategia de Desarrollo Local) por parte de los actores e instituciones que constituyen dichos GAL, a partir de unos ejes de inversión previamente fijados desde la Unión Europea y desde los gobiernos nacionales y/o regionales.

La asimilación del enfoque del DTI por parte de Leader se sustancia fundamentalmente en la creación de los GAL, como ámbitos experimentales de reflexión y cooperación entre actores locales y entre éstos y las escalas superiores de gobierno. Se trata por tanto de estructuras de gobernanza territorial multinivel destinadas a llevar a cabo procesos de innovación social e institucional, que permitan "construir un proyecto de sociedad" (Observatorio Europeo Leader, 1997:13) y no sólo gestionar fondos públicos europeos, estatales y/o regionales.

La flexibilidad que ha mostrado Leader desde su creación se fundamenta en el hecho de que su implementación se ha adaptado tanto a los denominados territorios de ruralidad profunda, como a los más dinámicos y complejos territorios periurbanos. Estos últimos suponen un verdadero desafío para el método Leader, por cuanto que se trata de espacios hacia los que se difunde la complejidad urbana y de los que se hace un uso urbano, "como si de una ciudad se tratara" (Indovina, 2007:18).

Las áreas periurbanas se comportan así como las nuevas periferias de la ciudad regional, dispersa y fragmentada, como un tercer espacio donde se entremezclan el paisaje y el paisanaje propio del mundo rural preexistente, con las nuevas funciones y las nuevas poblaciones urbanas, sin que puedan establecerse límites claros entre ambas realidades (Vanier, 2000; Entrena, 2005).

Ante ese escenario, los GAL se han constituido en potenciales estructuras de gobernanza territorial donde actores locales autóctonos y nuevos habitantes de origen urbano pueden coincidir y diseñar de manera consensuada una estrategia, que permita el desarrollo de sus comarcas y el encaje de las mismas en las dinámicas de la región urbana en la que se insertan.

\section{BREVE CARACTERIZACIÓN DE LA COMARCA DE LAS VEGAS. LA CUESTIONABLE DELIMITACIÓN DE UN TERRITORIO COMPLEJO}

La comarca de Las Vegas es un amplio territorio de $1.377 \mathrm{kms}^{2}$, esto es el 17,1\% del total regional, ubicado en el extremo suroriental de la Comunidad Autónoma de Madrid. Está compuesta por 23 municipios en los que, según datos del año 2010, residen algo más de 150.000 habitantes, es decir, el 2,3\% de los más de 6,4 millones de habitantes con que cuenta la región. Este territorio ha sido históricamente un espacio agrario debido a la presencia de las vegas de tres de los ríos más importantes que discurren por la Comunidad: los ríos Tajo, Tajuña y Jarama (figura 2). 
Figura 2: Localización de la comarca de Las Vegas al sureste de la Comunidad Autónoma de Madrid
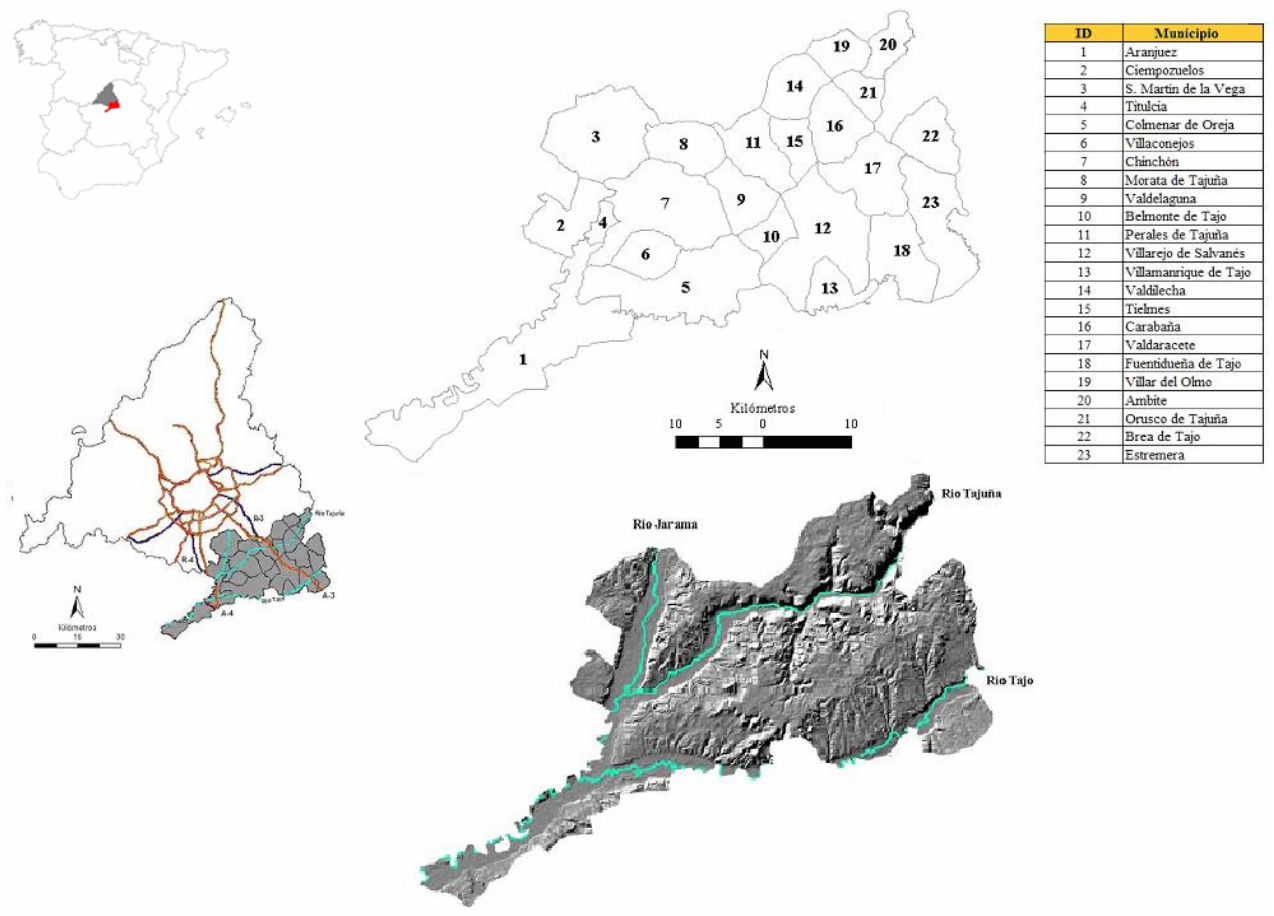

Fuente: Elaboración propia.

Cuando en 1995 se han de delimitar los contornos de esta comarca para la aplicación de la segunda fase de la iniciativa Leader, se va a utilizar como base de la misma la comarca agrícola preexistente, vertebrada alrededor de los tres ríos citados más arriba (MAPA, 1996; Alier et al., 2002). La elección de ese criterio sectorial valoraba ante todo la función que como despensa agraria de Madrid había jugado este espacio hasta los años cincuenta del siglo XX, obviándose, o al menos no considerando convenientemente, factores territoriales que hubiesen ofrecido una valoración más acertada de la articulación de la comarca en el funcionamiento del sistema socioeconómico regional de finales del siglo XX.

Un sistema regional que se ha caracterizado por lo que Castells (1985:49) definió como una "concentración descentralizada", es decir, por un doble proceso selectivo de concentración y difusión territoriales de actividades, funciones y población sobre el territorio de la creciente región urbana madrileña. Las grandes infraestructuras de transporte y comunicación radiales y orbitales se han convertido en la arquitectura que ha dado forma y que sostiene dichas dinámicas (Méndez, 2001; Méndez et al., 2010). 
Figura 3. Distribución de la población en la comarca de Las Vegas según sectores territoriales y municipios, 1991-2010

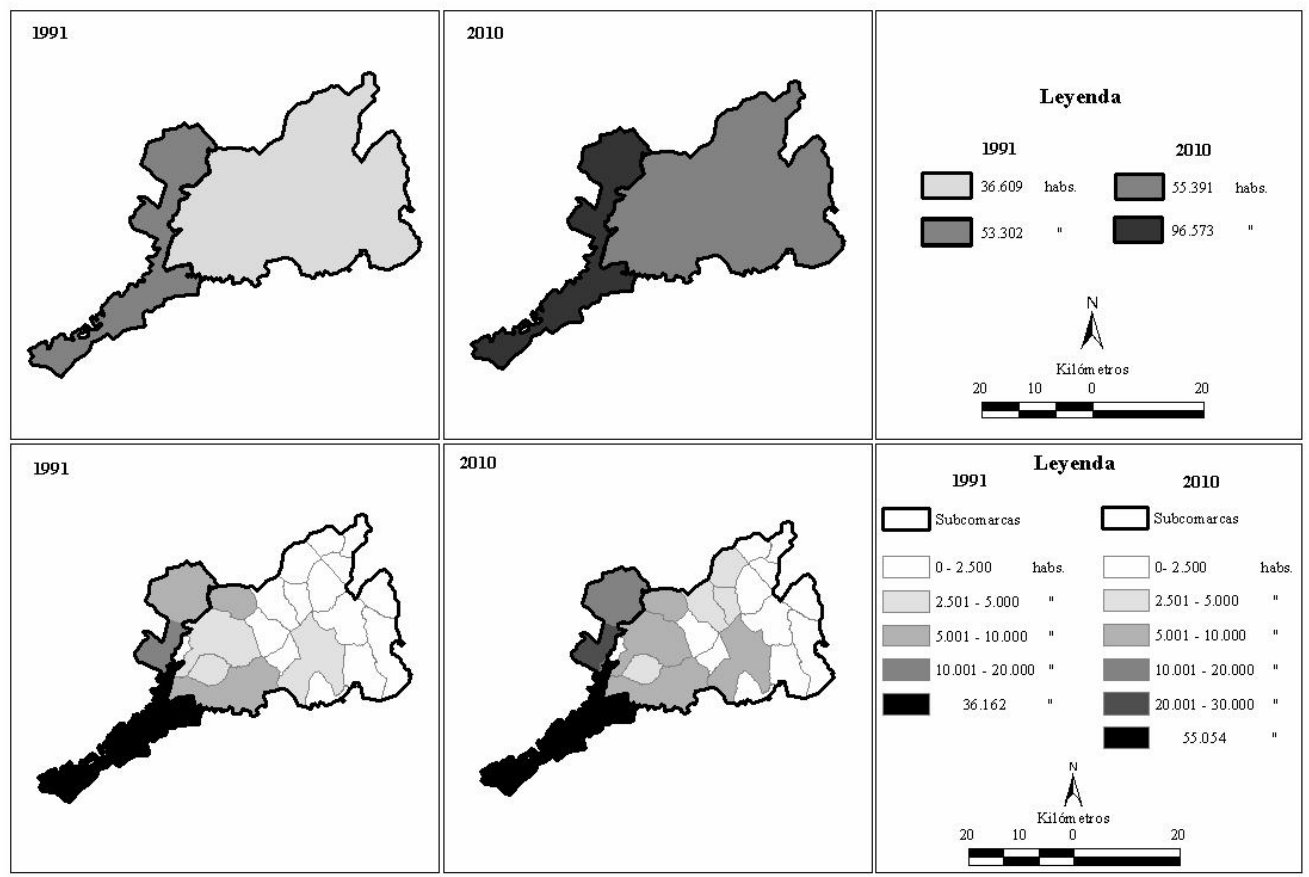

Fuente: Elaboración propia

Por ello, la elección de los tres ríos anteriormente citados como elementos vertebradores de este territorio periurbano, frente a los principales ejes de comunicación radiales que la atraviesan y que la conectan con el centro de la región funcional, dará lugar a la existencia de una dinámica territorial dual en la comarca. Dicha dualidad se manifiesta en la conformación de un área o sector occidental, compuesto por los municipios de Aranjuez, Ciempozuelos y San Martín de la Vega, y un sector oriental formado por el resto de los municipios.

El sector occidental, que se articula a lo largo del potente eje económico formado por la autovía estatal A-4, se comporta como un espacio metropolitano, tal y como reconocen diversos documentos oficiales de la administración regional, mientras que el sector oriental presenta una dinámica más ajustada a un espacio de expansión periurbana, como también indican esos mismos documentos oficiales (Consejería de Economía e Innovación Tecnológica, 2005; Consejería de Medio Ambiente, Vivienda y Ordenación del Territorio, 2010).

Varios indicadores muestran cómo a lo largo de las últimas décadas esa división interna no sólo no se ha reducido, sino que ha tendido a incrementarse. En este sentido, la dinámica demográfica reciente muestra que entre 1991 y 2010 el 70,2\% de los 62.127 nuevos habitantes que han llegado a Las Vegas, se han asentado en alguno 
de los tres municipios del sector occidental (figura 3). De esta manera, en 2010, de los 151.964 habitantes de la comarca, el 63,5\% residen en alguno de esos tres núcleos de población. En 1991, el porcentaje era del 58,9\%.

En cuanto a la dinámica económica reciente, un somero análisis de la evolución de empresas y trabajadores residentes a partir de los datos ofrecidos por la Tesorería General de la Seguridad Social (TGSS), para la década comprendida entre los años 2000 y 2009, y referidos a empresas y trabajadores afiliados según la Clasificación Nacional de Actividades Económicas a dos dígitos (CNAE-93 y CNAE-09, respectivamente), incide en la dualización de este territorio.

En este sentido, se ha realizado una clasificación funcional de las actividades económicas superadora de la tradicional división sectorial. La consideración de este tipo de clasificación tiene como objetivo desentrañar la complejidad inherente al proceso de crecimiento y diversificación de las actividades y empleos enmarcados en los sector de los servicios (Halbert, 2005, Méndez, et al., 2010 y 2011), proceso que afecta de manera más intensa a las regiones urbanas y a sus áreas de expansión.

De este modo, y partiendo de las clasificaciones estadísticas anteriormente citadas, se han agrupado las actividades económicas en torno a cuatro tipo de funciones. La primera de ellas aglutina las actividades dedicadas a la producción propiamente dicha, mientras que los otros tres grupos reúnen actividades de servicio en función del tipo de agente principal cuyas necesidades pretenden cubrir, ya se trate de las empresas o de la población, diferenciando en el caso de esta última cuando se comporta como usuaria de servicios públicos o como consumidora:

- Función de Producción: engloba aquellas actividades que aseguran la producción de bienes tangibles (agricultura, industria, construcción).

- Función de Intermediación (servicios a las empresas): agrupa aquellos servicios empresariales que con anterioridad o posterioridad a la producción material aportan un valor añadido a ésta y facilitan su circulación (dirección y gestión; finanzas; seguros; servicios jurídicos; publicidad y márketing, distribución, etc.), y aquellas otras actividades que sirven de apoyo a las empresas.

- Función Social (servicios a la población): aglutina por un lado los servicios destinados a la gestión de la res pública, y por otro los servicios dirigidos a satisfacer necesidades sociales relacionadas con la salud, la educación o los servicios sociales, todos ellos cubiertos tradicionalmente por la administración pública, pero que en los últimos años, y de manera significativa en la Comunidad de Madrid, tienden a ser progresivamente privatizados.

- Función de Consumo (servicios al consumidor): recoge todas aquellas actividades orientadas al consumo (comercio, hostelería, etc.), y aquellas vinculadas con los servicios personales. 
En el caso concreto de la región de Madrid, el sector de los servicios aglutinaba en 2009 al $85,3 \%$ del total de las empresas y al $82,4 \%$ de la mano de obra, mientras que en la comarca de Las Vegas este sector suponía el 69,6\% del tejido empresarial y el $68,5 \%$ de los trabajadores. En el interior de la comarca se observa en cualquier caso una diferenciación entre las áreas occidental y oriental. Así, en la primera de ellas el peso de las empresas de servicios en 2009 era del 75,7\%, mientras que en la zona oriental ese porcentaje se reducía al $59,8 \%{ }^{1}$; la mano de obra empleada en los servicios para ese mismo año era del $73,5 \%$ en el occidente de Las Vegas y del $58,6 \%$ en el sector oriental ${ }^{2}$.

Atendiendo a la clasificación funcional realizada, se observa que las estructuras empresariales de ambas sub-unidades pivotan en torno a funciones distintas. Mientras el perfil empresarial de la zona occidental está marcado por el creciente peso de las actividades de consumo, en el de la zona oriental, aun cuando las empresas de servicios son actualmente mayoritarias, las actividades de producción siguen teniendo un importante peso en el sistema productivo local.

De esta manera, la figura 4 muestra como durante el periodo considerado el perfil dibujado por la estructura empresarial del occidente comarcal ha tendido a aproximarse al dibujado por el conjunto de la región urbana, mientras que el del sector oriental se halla todavía en una fase de menor evolución, por el peso que aún tienen las empresas de construcción, industriales y agrarias en la zona.

En lo que se refiere a la estructura laboral, cabe destacar el pronunciado descenso que tanto en el área occidental como en el oriental han experimentado los trabajadores vinculados a las tareas de producción. Sin embargo, mientras en el sector oriental de Las Vegas este tipo de trabajadores siguen teniendo un peso significativo en su estructura laboral, en el sector occidental el perfil funcional de la mano de obra se ha desplazado desde las tareas de producción hacia las actividades de servicio, siendo especialmente significativo el crecimiento reciente de los trabajadores vinculados a los servicios de tipo social y de intermediación.

Esta evolución en la estructura de la mano de obra residente en la zona occidental de Las Vegas responde a dos procesos. Primero, a la consolidación de la ciudad de Aranjuez como nodo de servicios sociales en el sur de la región urbana, relacionado con actividades sanitarias, educativas y con servicios públicos generales (Hacienda, Seguridad Social, etc.); segundo, por el asentamiento reciente de trabajadores pendulares empleados en el centro de la región en actividades de intermediación.

\footnotetext{
${ }^{1}$ La distribución del resto de empresas según sectores de actividad en el año 2009 es: en el área occidental de la comarca: 1,9\% agrario; 9,1\% industria; y 13,3\% construcción. En el área oriental: 9,3\% agrario; $11,5 \%$ industria; y 19,4\% construcción.

${ }^{2}$ La distribución del resto de trabajadores según sectores de actividad en el año 2009 es: en el área occidental: 1,7\% agrario; $15,9 \%$ industria; y 8,9\% construcción. En el área oriental: 7,7\% agrario; $16,8 \%$ industria; y 17,0\% construcción.
} 
Figura 4. Evolución del perfil funcional de los tejidos empresarial y laboral en los sectores territoriales occidental y oriental de la comarca de Las Vegas y en la Comunidad de Madrid, 2000-2009 (en \%)

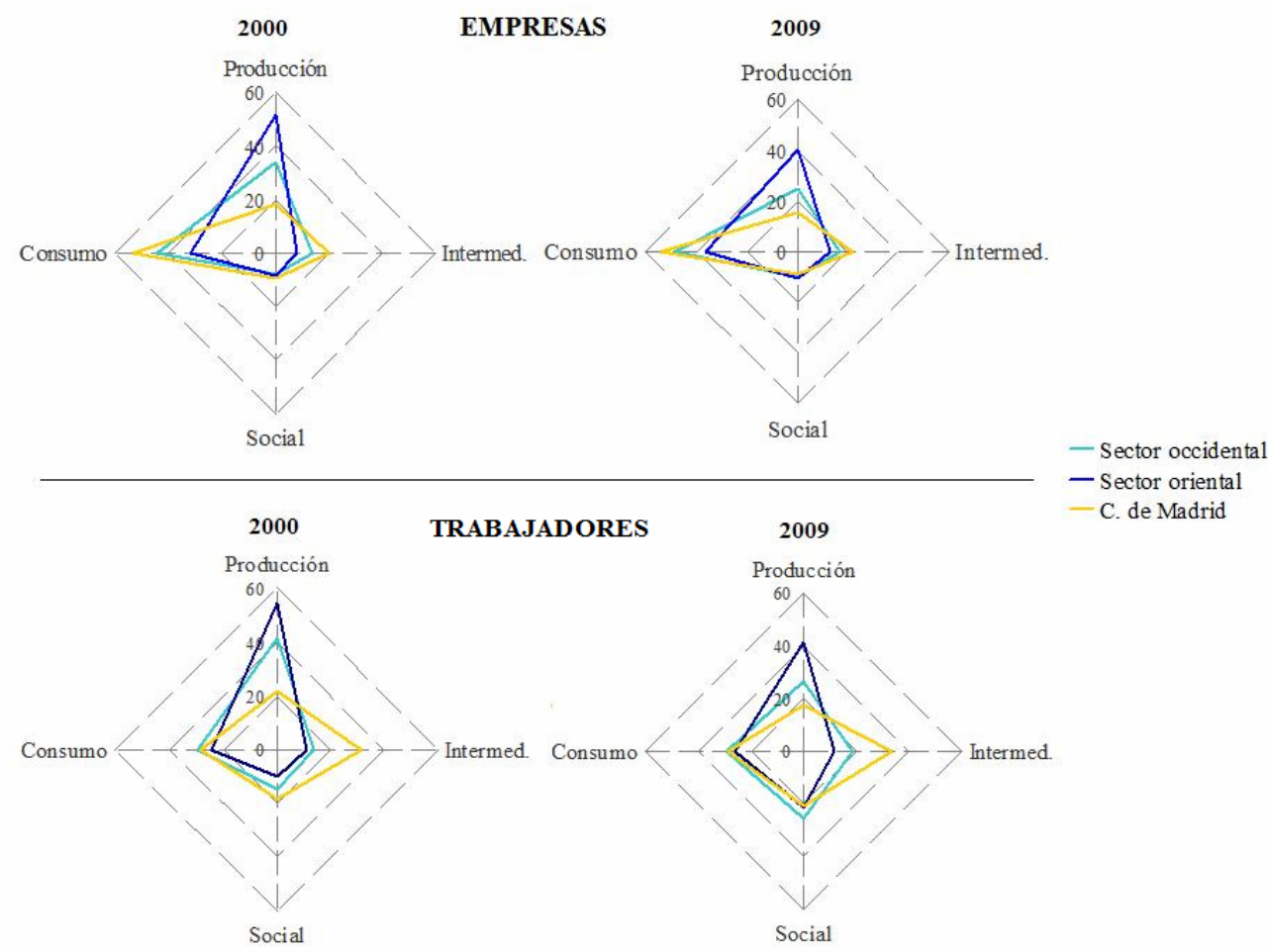

Fuente: Elaboración propia, a partir de la Tesorería General de la Seguridad Social

La presentación de estos breves datos estadísticos, que reflejan la dinámica demográfica, social y funcional experimentada en la región urbana madrileña durante las últimas décadas, cuestionan la validez de la delimitación de esta comarca Leader periurbana a partir de criterios agraristas, basados en la idealización de este territorio como antigua despensa agraria de Madrid.

En este sentido, el Observatorio Europeo Leader (1997:24) recomendaba diseñar las comarcas valorando cuestiones como "la cohesión social interna, una historia y tradiciones comunes y un sentimiento de identidad compartido que diesen forma a una verdadera unidad territorial rural". Los responsables regionales encargados de delimitar esta comarca Leader, consideraron que la presencia histórica sobre este territorio de una agricultura de vega tradicional podría actuar como elemento de cohesión social e identitaria. Sin embargo, el sector agrícola fue desarticulado en su mayor parte en la segunda mitad del siglo XX; por otro lado, este sector nunca actuó como nexo identitario entre las distintas comunidades que ocuparon este espacio, ya 
que los ríos que lo atraviesan, al no ser navegables, nunca han cumplido un papel vertebrador del territorio (Abad, 2013).

La formación de la actual región urbana madrileña ha reafirmado la debilidad de esos vínculos intermunicipales, así como el peso de las áreas centrales en la organización social y económica de esta comarca madrileña. En ese escenario tan complejo es en el que Aracove ha tenido que desenvolverse durante los últimos quince años como espacio experimental de gobernanza y desarrollo.

\section{EL GRUPO DE ACCIÓN LOCAL ARACOVE. CARACTERIZACIÓN BÁSICA Y PAPEL EN LA GOBERNANZA DE LA COMARCA DE LAS VEGAS}

\subsection{EL GRUPO DE ACCIÓN LOCAL ARACOVE: CARÁCTERÍSTICAS GENERALES, COMPOSICIÓN, ESTRUCTURA Y LIDERAZGO}

El GAL Aracove se constituyó el 29 de septiembre de 1995 (Consejería de Presidencia, Justicia e Interior, 2011), ex profeso para la gestión de la iniciativa comunitaria Leader II, a iniciativa de la Consejería de Economía del gobierno regional, a través de la Dirección General de Agricultura, bajo la forma jurídica de una asociación sin ánimo de lucro y personalidad jurídica propia.

La finalidad última que persigue Aracove es el establecimiento de un modelo de desarrollo rural sostenible y armónico en la comarca de Las Vegas. Para la consecución de esta meta el GAL ha implementado diversos programas desde su origen; programas europeos, estatales y regionales que pueden ser agrupados en torno a cuatro grandes líneas temáticas:

- Desarrollo y cooperación territoriales: Iniciativa europea Leader (19962006); Estrategia europea Leader (2007-2013); Programa estatal Proder; Programa Red Rural Nacional

- Empleo, Formación y Emprendimiento: Iniciativa europea Equal; Programa Regional Experimental de Empleo; Programa Regional de Orientación Profesional para el Empleo y asistencia para el Autoempleo (OPEA); Programa Regional Escuela Taller; Programa Regional Taller de Empleo; Red Regional Hiedra para mujeres emprendedoras.

- Nuevas Tecnologías: Programa Regional de Centros de Acceso Público a Internet (Red CAPI); Programa Regional de Centros de Difusión de la Innovación (Red CDI); Plan estatal Avanza2

- Otros

En conjunto, y entre 1997 y hasta 2012, Aracove ha gestionado un total de 50,5 millones de euros, de lo cuales el 51,5\% corresponden a la línea de Desarrollo y Cooperación Territoriales (26 millones de $€$ ); el 30,6\% a la de Nuevas Tecnologías (15,5 millones de $€)$; el 17,6\% a la línea de Empleo, Formación y Emprendimiento 
(8,9 millones de $€)$; y un exiguo $0,2 \%$ corresponde a Otros proyectos $(0,1$ millones de $€)$.

En cuanto a su composición interna, en 2011 Aracove estaba integrado por 62 socios, de los cuales 24 eran instituciones públicas y 38 entidades privadas. Entre las instituciones públicas, además de los 23 ayuntamientos de la comarca se encuentra el organismo regional Consejo Regulador de la Denominación de Origen "Vinos de Madrid"; mientras que entre los socios privados 33 son empresas (16 pertenecen al sector agroindustrial, 15 al sector del turismo, del ocio y de la hostelería, y 2 al sector financiero); 4 son asociaciones empresariales (3 agrarias y 1 turística); y 1 es un sindicato agrario. Para poder formar parte de este GAL, todos estos socios aportan una cuota económica anual de carácter simbólico.

La organización interna de Aracove es similar a la de otros GAL, dado que cuenta con un órgano de representación, la Asamblea General; un órgano de gobierno, la Junta Directiva; y un tercer órgano dedicado a la gestión y a la administración técnica: el Equipo Técnico de Gestión.

A partir de esta estructura interna se organizan los liderazgos en Aracove, cuestión que resulta fundamental para determinar el grado de éxito en iniciativas como ésta. En ese sentido, se pueden determinar tres tipos de liderazgo que hasta la fecha se han complementado entre sí, lo que ha evitado conflictos al interior del grupo por el control del mismo.

El primero de esos liderazgos hace referencia a la administración pública local, que aunque ostenta un menor número de representantes que el sector privado en la Junta Directiva, en cumplimiento de la normativa europea, dichos cargos son los de mayor peso institucional y de dirección del grupo (Presidente; Vice-Presidente; Secretario y Tesorero). Esta situación se viene produciendo desde el origen de Aracove, momento en el que se llegó a un acuerdo tácito para que los ayuntamientos ocupasen siempre estos cargos, y además para que siempre lo hicieran los mismos ayuntamientos.

Como se indica, la mayor parte de los socios justifican el control directo del grupo por parte de las administraciones públicas locales, aduciendo que, frente al sector privado, los representantes públicos gozan de un mayor respaldo legal, de una mayor capacidad de interlocución interadministrativa, de una mayor capacidad financiera para acometer proyectos que requieren fuertes inversiones, así como de la legitimidad democrática que aportan las urnas. Se considera también que esas tareas de representación forman parte de la labor, de los horarios y de los honorarios de los electos. En definitiva, se acepta ampliamente el liderazgo de los ayuntamientos por cuanto que se entiende es consustancial a su naturaleza de administración pública.

El segundo de los liderazgos que se manifiesta a través de la organización interna de Aracove es el del Equipo Técnico y de Gestión, que cuenta con un alto grado de valoración entre los socios del grupo, así como también entre algunos actores territoriales externos al GAL que conocen el alcance de sus actividades. Se llega incluso a producir una cierta confusión en algunos de ellos, pues asimilan la parte con el todo, es decir, que identifican a Aracove sólo con el equipo técnico. En este sentido, es esencialmente valorada su contribución a la reducción de la burocracia y 
de las complejidades inherentes a la solicitud de ayudas públicas. De esta manera, la influencia de la gerencia técnica se apoya esencialmente en lo que hace -bien-, más que en lo qué es o representa dentro del organigrama de Aracove.

Finalmente, existe un tercer liderazgo no bien reflejado en la estructura interna del grupo, pero cuya incidencia en los discursos y en las actuaciones de Aracove es manifiesta. Se trata del liderazgo "blando" ejercido por el sector agroindustrial de la zona, en especial, por el subsector de las cooperativas y principalmente por el subsector del vino, de gran tradición en la comarca. Ambos se estructuran alrededor de dos entidades de referencia: la Unión de Cooperativas Agrarias de Madrid (UCAM) y el Consejo Regulador de la Denominación de Origen "Vinos de Madrid" respectivamente.

Su capacidad de influencia dentro de Aracove se deriva precisamente de su mayor articulación y del hecho de que tanto UCAM como el Consejo Regulador tienen un carácter regional, lo que les obliga a ubicar su sede principal en la ciudad de Madrid, desde donde han tejido densas redes de relaciones con instituciones y organismos de poder y de información, tanto estatales como regionales, radicadas también en la capital.

Desde esa posición impulsan su visión sobre lo qué es y debe de hacer Aracove como GAL. Así, consideran que Aracove, a través de su gerencia técnica, debe centrar su trabajo en la búsqueda de financiación pública para redirigirla principalmente hacia el sector empresarial de la zona, pues entienden que ésta es la única manera posible de lograr el desarrollo económico de la comarca. Ambas entidades sostienen la necesidad de profundizar este modelo gerencialista en los próximos años, dotando a Aracove de una mayor profesionalidad empresarial y reduciendo progresivamente el papel que hasta ahora ha jugado el sector público en la dirección del grupo, de tal forma que la función de las administraciones locales pase a ser fundamentalmente la de representación institucional de Aracove.

Este modelo de gestión empresarial de la gobernanza es asumido por gran parte de la administración pública local y por la gerencia del grupo.

En definitiva, la existencia de estos tres liderazgos complementarios supone la materialización de un proceso de "captura de la red de cooperación", que es lo que en principio debía ser Aracove como GAL, por parte del sector agroindustrial y de la administración local, con la aquiescencia de la gerencia técnica.

\subsection{LA ADMINISTRACIÓN REGIONAL COMO AGENTE CLAVE EN EL DISEÑO DEL MODELO DE GOBERNANZA EMPRESARIAL APLICADO POR ARACOVE}

La construcción y asimilación de este discurso empresarial/gerencialista de la gobernanza no se debe, en cualquier caso, al liderazgo "blando" ejercido desde el sector agroindustrial de la zona, ya que dicho liderazgo es más bien una de las consecuencias que se derivan de un conjunto de procesos vinculados a la implantación de Leader en la región y en la comarca. 
En este sentido, la creación de los GAL madrileños se produce a mediados de los años noventa coincidiendo con un cambio de signo político en el gobierno regional, que virará hacia posiciones conservadoras liberales. Esta evolución en la política regional ha tenido incidencia en la construcción de determinados discursos y prácticas llevadas a cabo por el conjunto de GAL que operan en las áreas periurbanas de la Comunidad de Madrid, entre ellos Aracove. Esto ha sido así porque el cambio de orientación política en la administración regional se produjo justo en el momento en el que ésta debía fomentar la creación ex novo de GAL, para implementar la segunda fase de Leader $^{3}$ en la Comunidad, de tal forma que el giro político impulsó una concepción agrarista de Leader y una visión empresarial de su gestión multinivel (región-local).

Ese modelo de gestión empresarial se sustentó en el peso que dentro de la nueva administración regional adquirió el Departamento de Proyectos y Planificación Rural de la Escuela Técnica Superior de Ingenieros Agrónomos de la Universidad Politécnica de Madrid. Algunos de sus miembros más destacados ocuparon cargos políticos de primer nivel en la nueva administración conservadora, desde donde trazaron las líneas maestras que van a definir a los GAL regionales desde entonces, además de ocupar las gerencias y equipos técnicos de estos GAL con ingenieros agrónomos pertenecientes o vinculados de alguna manera con dicho equipo universitario.

En lo que se refiere a la aplicación de un modelo de gobernanza gerencial por parte de los GAL madrileños, éste queda sintetizado en las palabras de los ingenieros responsables de su diseño al afirmar que:

... la conducción de un proyecto de desarrollo territorial presenta muchas analogías con la conducción de un proyecto en la empresa. Por esta razón, los métodos que se utilizan en el mundo empresarial pueden ser útiles a los responsables de los Grupos de Acción Local (AEIDL, 1995). Sin embargo estas analogías tienen sus límites: los productos, los clientes, los beneficiarios de la acción del Grupo de Acción Local no se pueden comparar plenamente con los de una empresa [...]. Por ello, el Grupo de Acción Local debe innovar en sus prácticas, tanto para adaptar los métodos empresariales a los objetivos del desarrollo, como para asegurarse que este prosigue correctamente su objetivo principal, que es el de crear una dinámica de desarrollo territorial duradera (Alier et al., 2005).

${ }^{3}$ Los GAL, como estructuras experimentales de gobernanza, surgen durante la segunda fase de Leader por indicación de la Comisión Europea. En la primera etapa, que se desarrolló entre 1991 y 1994, las administraciones regionales se sirvieron en la mayor parte de los casos de entidades públicas y privadas preexistentes para delegar en ellas la gestión de la iniciativa. Así, el único Leader I que se desarrolló en la Comunidad de Madrid, en concreto en la comarca de la Sierra Norte, fue gestionado por el Patronato Madrileño de Areas de Montaña, un organismo público de carácter autónomo, dependiente del gobierno regional (Troitiño et al., 2006) 
Tabla 1. Características generales de los partenariados de gestión y animación

\begin{tabular}{|c|c|c|}
\hline & GESTIÓN & ANIMACIÓN \\
\hline Fines y Objetivos & $\begin{array}{c}\text { Obtener, repartir y } \\
\text { consumir recursos financieros }\end{array}$ & $\begin{array}{l}\text { Identificar y movilizar a los } \\
\text { actores locales }\end{array}$ \\
\hline $\begin{array}{c}\text { Dinámicas } \\
Y \\
\text { Lógicas }\end{array}$ & $\begin{array}{c}\text { Lógica tecno-económica } \\
\text { Oportunidad coyuntural } \\
\text { Posibilidad de obtener } \\
\text { financiación. } \\
\text { Presentar informes } \\
\text { elegibles en un procedimiento } \\
\text { burocrático }\end{array}$ & $\begin{array}{l}\text { Lógica socio-institucional } \\
\text { Cambio estructural } \\
\text { Construir un proyecto de } \\
\text { sociedad. } \\
\text { Pedagogia y movilización } \\
\text { que requieren tiempo }\end{array}$ \\
\hline Resultados esperados & $\begin{array}{c}\text { Realización de un proyecto } \\
\text { oprograma }\end{array}$ & $\begin{array}{l}\text { Innowación y dinamización } \\
\text { del territorio }\end{array}$ \\
\hline $\begin{array}{c}\text { Composición } \\
y \\
\text { Apertura }\end{array}$ & $\begin{array}{l}\text { Partenariado cerrado o } \\
\text { capturado por unos pocos } \\
\text { actores con intereses } \\
\text { económicos }\end{array}$ & $\begin{array}{c}\text { Partenariado abierto al } \\
\text { mayor número de entidades } \\
\text { territoriales posibles, entomo a } \\
\text { la discusión de ideas y temas } \\
\text { ternitoriales }\end{array}$ \\
\hline
\end{tabular}

Fuente: Adaptado de Observatorio Europeo Leader, 1997

Abundando sobre en esta idea, los ingenieros responsables de la planificación de Leader en la Comunidad de Madrid defienden la orientación por proyectos como estrategia de gestión para fomentar la cooperación social en el desarrollo, al argumentar que:

[...] los planificadores en el ámbito público deben situarse como profesionales que se responsabilicen de los procesos que presiden y de las acciones que acometen, adoptando una misión nueva y más emprendedora que se ha denominado función empresarial. Estos empresarios de la planificación son principalmente movilizadores de los recursos, que buscan concertar las energías públicas y privadas en soluciones innovadoras para los desafiantes problemas del dominio público. Estas coordenadas de fondo y estos valores del planificador están íntimamente relacionados con el proceso que, en la bibliografía internacional, se ha definido como aprendizaje social [...]. La aplicación de la orientación por proyectos -Management by project-, más extendido en el ámbito empresarial, junto con metodologías basadas en el aprendizaje 
social, permitirá adoptar planteamientos novedosos en la planificación en el ámbito público y al mismo tiempo enriquece el cuerpo de doctrina de la dirección y gestión de proyectos. (Cazorla et al., 2001)

Para materializar esta idea se dotó a la gerencia de los GAL de una dimensión exclusivamente técnica, tal y como exponen Cazorla et al. (2005:703) al asegurar que la misión de este órgano "era la más cercana a la población local y consistía en dar apoyo técnico a los promotores de proyectos, elaborar expedientes e informes y dar seguimiento a los proyectos". En ningún momento se concibió que este órgano técnico actuase como un agente de animación territorial destinado a "construir un proyecto de sociedad" (Observatorio Europeo Leader, 1997:13), involucrando en ese proceso a los principales actores territoriales de sus respectivas comarcas (Tabla 1).

Por este motivo, los equipos técnicos de los GAL madrileños no se concibieron para dinamizar y movilizar los territorios, a partir de la realización de una intensa labor pedagógica entre la población y los actores económicos y políticos locales, sino como organismos externos, pero dependientes, de la administración regional, para informar sobre las ayudas públicas y gestionar técnicamente los procesos inherentes a su tramitación.

En este sentido, conviene indicar que esa visión técnica y gerencial de los GAL ha conllevado una progresiva dependencia económica y técnica de los mismos respecto del gobierno regional, ya que los mecanismos de gestión de las ayudas diseñados en las sucesivas fases de la iniciativa Leader han incrementado los procesos de burocratización y de control. Inicialmente el sometimiento de estas entidades a la decisión de la administración regional era sutil, pues los técnicos de la Comunidad emitían un informe de elegilibilidad, acompañado de una serie de recomendaciones; ese informe no era vinculante, pero sí era orientativo. En la actualidad, el control es más directo, por cuanto que el informe de los técnicos de la administración regional es vinculante y determina por tanto la decisión final de los GAL, de tal forma que al perder la capacidad de gestionar directamente los fondos Leader, los GAL madrileños han perdido uno de sus principales valores: la proximidad al territorio.

El modelo gerencial propuesto ha convertido así a los GAL madrileños en ventanillas -externalizadas- de gestión de ayudas públicas, lo que ha generado expectativas económicas entre los actores económicos y políticos locales mejor organizados e informados. Estos grupos de poder han "capturado" la organización de los GAL, bien copando los principales puestos ejecutivos y de dirección, bien situándose en una posición de influencia, tal y como ha sucedido en Aracove. A medida que estos grupos de presión han ido asentando su poder en la estructura interna de los GAL, la ciudadanía se ha ido distanciando de ellos al percibirlos como entidades al servicio de intereses creados, y no como partenariados locales destinados a la construcción conjunta de un proyecto territorial común. 


\subsection{LA INCOMPRENSIÓN DE LA FILOSOFÍA TERRITORIAL DE LEADER POR PARTE DE LOS AGENTES INTEGRADOS EN ARACOVE}

En el caso concreto de Aracove, los procesos regionales descritos más arriba se han visto acompañados por una serie de procesos locales que han facilitado el asentamiento del modelo de gestión empresarial como modus operandi de este grupo.

En este sentido, aunque Aracove se constituyó en 1995 no comenzó su actividad hasta 1997 por desavenencias entre los altos cargos de la Dirección General de Agricultura y los ayuntamientos de la comarca, respecto a la forma y a las personas que debían asumir la responsabilidad técnica y la gerencia del GAL durante Leader II. El retraso en la designación de estas personas supuso que Aracove se saltase la etapa inicial de Adquisición de Capacidades, una etapa clave para la comprensión de la filosofía Leader.

Dicha etapa fue diseñada por la Comisión Europea para capacitar a los técnicos de aquellos grupos que, como Aracove, iniciaban su trabajo en esta segunda fase de Leader y que, además, carecían de una experiencia previa en la gestión de proyectos de desarrollo bajo el enfoque territorial e integrado de la iniciativa. De este modo, el equipo técnico del grupo inició la gestión de Leader II sin haber pasado por la etapa de capacitación previa.

Por otra parte, este conjunto de circunstancias - retraso en la puesta en marcha del equipo técnico; no aplicación de la fase de Adquisición de Capacidades; inexistencia de experiencias de desarrollo territorial e integrado anteriores - también han conllevado la ausencia de un periodo de reflexión conjunta entre los socios y técnicos de Aracove y la sociedad comarcal en torno a qué elementos y valores se podrían estructurar una identidad compartida.

Por sus características, esta comarca presenta tres factores que dificultan la aparición de un sentimiento de pertenencia a la misma:

- Se trata de una comarca sin raíz histórica, esto es, que se apoye en la existencia de potentes vínculos e instituciones tradiciones compartidas por las comunidades humanas que lo ocupan. De esta forma, en la zona ha evolucionado un importante sentimiento localista que dificulta la puesta en marcha de proyectos de cooperación municipal.

- Relacionado con lo anterior, las investigaciones llevadas a cabo por Utanda (1996 y 2004) demuestran el peso que sobre este territorio ha tenido la gran propiedad en manos de una pequeña clase terrateniente, frente a formas de propiedad y de gestión comunal de la tierra. En base a esta realidad, se ha desarrollado entre la población autóctona un intenso sentimiento hacia la propiedad privada y hacia el "terruño", a partir del cual toman forma comportamientos individualistas y situaciones de desconfianza, que han frenado la aparición de una cultura de cooperación y colaboración ciudadanas.

- Finalmente, la influencia de Madrid y su área metropolitana ha desarticulado las escasas formas locales de organización y las centralidades tradicionales, sometiendo a este territorio a su creciente influencia. En este sentido, y tal y 
como se indicó unas líneas más arriba, la comarca de Las Vegas, y en especial su área más occidental, se integra en la actual región urbana como espacio residencial dirigido a una parte de las clases medias urbanas; clases medias cuya implicación en el desarrollo de este territorio periurbano es mínimo, por cuanto que no llegan a desarrollar vínculos estrechos con el mismo.

Por tanto, se puede afirmar que el principal déficit que arrastra Aracove desde su origen es precisamente el no haber llevado a cabo una intensa labor de dinamización sociocultural dirigida a superar las limitaciones señaladas. Al no haber acometido esa difícil tarea de animación territorial, y haber centrando su actividad únicamente en la gestión de programas y fondos públicos, el GAL se encuentra hoy con que gran parte de los agentes económicos, sociales y civiles de la comarca desconocen no sólo a qué se dedica Aracove, sino incluso su propia existencia. De este modo, la transformación de Aracove como la entidad de referencia para la gobernanza territorial de la comarca se ha visto dificultada y limitada.

Por otro lado, durante los últimos años se ha generando entre una parte de la sociedad y de los agentes políticos y económicos comarcales un sentimiento de abandono institucional, que se apoya en la ausencia de intervenciones por parte de la administración regional dirigidas a la dinamización económica de este territorio. A excepción de las inversiones gestionadas a través de Aracove, Las Vegas apenas ha sido objeto de inversión pública.

Alrededor de este sentimiento de abandono institucional están apareciendo iniciativas desde distintos ámbitos y con diferentes enfoques y grados de madurez, que pretenden articular y movilizar a actores e instituciones diversas en la búsqueda del desarrollo económico y social del sureste madrileño.

La aparición de estas iniciativas supone un fracaso para Aracove porque reflejan su incapacidad para anticiparse a ese sentimiento de abandono, así como para liderar y articular la reacción de la zona frente al mismo. En este sentido, ni la propia existencia de Aracove, ni los diferentes programas financiados que ha gestionado durante los últimos 15 años son percibidos como intervenciones destinadas a desarrollar económica y socialmente este territorio, lo que evidencia la falta de liderazgo y de visibilidad del grupo en la comarca.

En resumen, la incorrecta interpretación que desde los orígenes de Aracove se ha hecho de la filosofía territorial inherente al método Leader ha implicado:

- que no se haya generado un proceso de cohesión social, a partir del cual se puedan construir los vínculos que cementen la adhesión de la sociedad, del empresariado y de las entidades públicas de la comarca, tanto al territorio delimitado como al proyecto de desarrollo diseñado para el mismo;

- que no se haya conseguido integrar a las asociaciones civiles en la actividad diaria de Aracove, así como tampoco a gran parte de su tejido empresarial. Esto ha hecho que el grupo quede capturado por los representantes políticos 
locales y por agentes económicos vinculados a la agroindustria tradicional local;

- que Aracove no sea considerado el espacio de referencia en materia de desarrollo y gobernanza territorial en la comarca de Las Vegas, y que, en torno a ese vacío, surjan ahora nuevas iniciativas civiles y empresariales con la intención de cubrirlo;

- y finalmente, que perviva un enfoque gerencial y técnico frente a un enfoque más territorial y social. El predominio de un modelo de gobernanza empresarial en el seno de Aracove ha supuesto que las inversiones asociadas a los distintos programas gestionados sean contemplados como un fin en sí mismo, y no como el medio para lograr metas más amplias y ambiciosas como cohesionar a la sociedad comarcal en torno al territorio compartido, o diseñar conjuntamente un proyecto de desarrollo para el mismo. Bajo este enfoque gerencial, todo lo más que ha conseguido Aracove ha sido financiar proyectos de inversión puntuales, sujetos a unas líneas de subvención prefijadas por las administraciones públicas europea y regional, y en ningún caso se puede afirmar que ha logrado un desarrollo global de Las Vegas, ni su adecuada inserción en la región urbano-funcional madrileña a la que pertenece.

\section{REFLEXIONES FINALES}

El éxito o el fracaso de Leader como herramienta para implantar un modelo de desarrollo territorial sostenible en los espacios rurales y periurbanos europeos, tiende a medirse sólo en función de variables cuantitativas tales como la cantidad de fondos públicos y privados invertidos o como el número de proyectos implementados. Sin embargo, a partir de la investigación realizada, se considera oportuno tomar en cuenta también otro tipo de factores de carácter más cualitativo, que permitan evaluar los logros obtenidos por los GAL en la dinamización de las comarcas sobre las que han actuado. Lo que al final determina la consecución o no del modelo de desarrollo territorial deseado es la capacidad de estas entidades para establecer procedimientos de participación activa de los principales agentes políticos, económicos y civiles presentes en sus comarcas, tanto en el diseño de las estrategias de desarrollo local como en procesos de cohesión territorial.

En este sentido, el presente artículo pone el acento en el quién hace y en el cómo hace del método Leader, frente a la cuestión del cuánto predominante hasta la fecha; y es ahí donde adquiere importancia la cuestión de la gobernanza y de los diferentes modelos en los que ésta se manifiesta, para analizar el éxito o el fracaso en la gestión de Leader como instrumento dirigido a la implantación de un modelo de desarrollo territorial sostenible para los espacios de expansión periurbanos en Europa.

Por la complejidad de las dinámicas económicas, sociales y funcionales de estos nuevos territorios de borde, creemos que los GAL que operan en ellos deben aplicar un modelo de gobernanza de tipo territorial, frente al modelo empresarial que ha predominado en el caso presentado en este artículo. El análisis del GAL Aracove 
demuestra que una correcta gestión técnica de los fondos asociados a la iniciativa Leader, o a cualquier otro programa similar, no garantiza per se los necesarios procesos de cohesión territorial interna y regional que reclaman este tipo de espacios.

Esto es así porque bajo los modelos empresariales de la gobernanza se considera un fin en sí mismo la correcta gestión financiera de los programas implementados, en la convicción de que esto generará por sí mismo el crecimiento de la actividad económica y, a partir de ese crecimiento, el desarrollo. Sin embargo, como se ha observado en el caso de la comarca de Las Vegas, bajo el modelo gerencial aplicado por Aracove el dinero público ha acabado diseminándose por el territorio en pequeños proyectos de inversión, con escasa capacidad para modificar las dinámicas generales que afectan a este territorio.

El modelo empresarial se demuestra así ineficiente. Al dotar de un carácter finalista a los programas públicos, olvida que la función principal de los mismos es la de servir como instrumentos para construir y/o consolidar un proyecto de desarrollo territorial más amplio, y no el mero reparto de las ayudas públicas entre unos pocos actores públicos y privados.

Por otra parte, el estudio de caso presentado también pone de manifiesto cómo la aplicación de un modelo empresarial por parte de los GAL, conlleva la captura de la red de cooperación, que se supone son estas entidades, por parte de los actores políticos y económicos con mayor poder, influencia e información en sus territorios. Las expectativas económicas generadas entre esos actores impilica que quieran posicionarse convenientemente para acceder en condiciones más ventajosas a la financiación pública asociada a los distintos programas gestionados. Además, como se ha observado en el caso concreto de Aracove, estos actores también ponen en marchan sutiles mecanismos para dificultar o desincentivar el acceso de otros agentes comarcales a la estructura interna de los GAL.

La captura de la red de cooperación conlleva a su vez el distanciamiento de estas entidades con respecto a la sociedad civil y al tejido empresarial que queda fuera del grupo. En el caso de la comarca de Las Vegas se ha observado como ese distanciamiento ha fomentado procesos de auto-organización por parte de los sectores más dinámicos de la población y del tejido empresarial comarcal no incluidos en Aracove, lo que ha incrementado aún más la distancia que los separa de este GAL.

Finalmente, el ejemplo de Aracove pone también de manifiesto la ineficacia del modelo empresarial de gestión de la gobernanza en lo que se refiere a las relaciones interescalares entre la estructura de gobernanza local y la estructura de gobierno regional, por cuanto que éstas se están llevando a cabo bajo principios de jerarquía y autoridad y no bajo principios de cooperación. De esta manera, el GAL Aracove ha acabado por comportarse como una agencia cuya actuación está subordinada a las directrices que emanan del gobierno regional, y no como su socio, como su partenaire, para, mediante un proceso de diálogo, determinar el mejor encaje del modelo territorial comarcal en las dinámicas territoriales de la región urbana madrileña. 


\section{BIBLIOGRÁFIA}

ABAD, L. D. (2013): Territorio, Cooperación y Desarrollo. La iniciativa europea Leader como modelo de gobernanza en espacios periurbanos. Dos casos de estudio en la Comunidad de Madrid: las comarcas de la Sierra Norte y de Las Vegas. Tesis Doctoral. 869 páginas. En prensa.

ALIER, J. L., DE LOS RIOS I., DÍAZ PUENTE, J. M. y YAGÜE, J. L. (2002): “La planificación del desarrollo en la iniciativa Leader Plus: Innovación en diversas regiones"; en De los Ríos Carmenado, I (coord.): Innovación para el desarrollo rural: la iniciativa Leader como laboratorio de aprendizaje. Comunidad de Madrid, pp. 107-138.

ALIER, J. L., DE LOS RÍOS, I., DÍAZ PUENTE, J. M. y YAGÜE, J. L. (2005): “La inciativa comunitaria LEADER: las claves del éxito en la gestión de proyectos de desarrollo". Comunicación presentada en el XVII Congreso Nacional de Ingeniería de Proyectos, organizado por la Asociación Española de Ingeniería de Proyectos en Murcia entre los días 19 y 21 de septiembre. Disponible en: http://www.unizar.es/aeipro/finder/ORGANIZACION\%20Y\%20DIRECCION/D G01.htm

ARTMANN, J., HUTTENLOHER, CH., KAWKA, R. y SCHOLZE, J. (2012): Partnership for sustainable rural-urban development: existing evidences. Executive Summary. Proyecto enmarcado en la Acción Europea RURUBAN. Disponible en: http://ec.europa.eu/regional_policy/what/cohesion/urban_rural/index_en.cfm

BOISIER, S. (2004): "Una (re)visión heterodoxa del desarrollo (territorial): un imperativo categórico"; en Estudios Sociales: Revista de Investigación Científica, Vol. $12, n^{\circ} 23$, pp. 9-36.

CASTELLS, M. (1985): "Reestructuración económica, revolución tecnológica y nueva organización del territorio"; en Echenagusía, J. (ed.): Metrópolis, territorio $y$ crisis. Asamblea de Madrid. Madrid.

CAZORLA, A., CANO, J. L. y DE LOS RÍOS, I. (2001): "La orientación por proyectos como estrategia de gestión para fomentar la cooperación social en el desarrollo" en Actas del XVII Congreso Nacional de Ingeniería de Proyectos, Universidad de Murcia, Septiembre, 2001. Disponible en: http://www.unizar.es/aeipro/finder/ORGANIZACION\%20Y\%20DIRECCION/D G02.htm

CAZORLA, A., DE LOS RÍOS, I y DÍAZ, J. M. (2005): "La iniciativa comunitaria LEADER como modelo de desarrollo rural: aplicación a la región capital de España"; en Agrociencia, vol. 39, n $^{\circ} 6$ noviembre-diciembre, pp. 697-708.

COMISIÓN EUROPEA (2010): Comunicación de la Comisión al Parlamento Europeo, al Consejo Europeo, al Comité Social y Económico Europeo y al Comité de las Regiones. La PAC en el horizonte 2020: responder a los retos futuros en el ámbito territorial, de los recursos naturales y alimentario. COM 2010 (672) final. Bruselas.

CONSEJERÍA DE ECONOMÍA E INNOVACIÓN TECNOLÓGICA (2005): Atlas Estadístico de la Comunidad de Madrid 2005, Madrid. 
CONSEJERÍA DE MEDIO AMBIENTE, VIVIENDA Y ORDENACIÓN DEL TERRITORIO (2010): Programa de Desarrollo Rural de la Comunidad de Madrid, 2007-2013. Volumen I. Dirección General de Medioambiente. Madrid.

CONSEJERÍA DE PRESIDENCIA, JUSTICIA E INTERIOR (2011): Resolución del Director General de Seguridad e Interior por la que se dispone la inscripción de la modificación de los Estatutos y de la Junta Directiva u órgano de representación de la entidad denominada ARACOVE (Asociación de Desarrollo Rural Aranjuez-Comarca de Las Vegas), Registro de Asociaciones, Madrid.

ENTRENA DURÁN, F. (2005): "Procesos de periurbanización y cambios en los modelos de ciudad. Un estudio europeo de casos sobre sus causas y consecuencias"; en Papers. Revista de Sociología, no 78, pp. 59-88.

EUROPEAN NETWORK FOR RURAL DEVELOPMENT (2013): “Contribución a los vínculos urbano rurales en las regiones periurbanas"; en ENRD Magazine, $\mathrm{n}^{\mathrm{o}}$ Invierno 2012/2013 (versión en español).

FARINÓS DASÍ, J. (2008): "Gobernanza territorial para el desarrollo sostenible: Estado de la cuestión y agenda"; en Boletín de la Asociación de Geógrafos Españoles, ${ }^{\circ} 46$, pp. 11-32.

GONZÁLEZ DE CANALES, F. (2011): Desarrollo Rural Territorial. Instituto de Desarrollo Comunitario. Disponible en: http://www.idcfederacion.org/es/images/stories/pdf/desarrollo-rural-territorial.pdf

HALBERT, L. (2005): "Les métropoles, moteurs de la dématerialisation du système productif urbain français: une lecture sectorielle et fonctionnelle (1982-1999)"; en Bulletin de l'Association des Géographes Français, vol. 83, n³, pp. 279-297.

INDOVINA, F. (2007): "Introducción: antes de la ciudad difusa"; en Indovina, F. (coord.): La ciudad de baja densidad. Lógicas, gestión y contención. Diputació de Barcelona. Xarxa de municipis. Colección Estudios. Serie Territorio, $\mathrm{n}^{\circ} 1$. Barcelona

JACQUIER, C. (2008): "La gouvernance urbaine: entre complications et complexités, comment s'orienter?". Comunicación presentada en el Coloquio: Hacia una nueva gobernanza de los territorios, organizado el 15 de septiembre de 2008 en Reims.

LE GALÈS, P. (1995): "Du gouvernement des villes à la gouvernance urbaine"; en Revue française de science politique, vol. 45, $\mathrm{n}^{\mathrm{o}}$ 1, pp. 57-95.

MÉNDEZ, R. (2001): "Transformaciones económicas y reorganización territorial en la región metropolitana de Madrid"; en EURE, Revista Latinoamericana de Estudios Urbano Regionales, vol. XXVII, $\mathrm{n}^{\mathrm{o}} 80$. http://redalyc.uaemex.mx/pdf/196/19608007.pdf

MÉNDEZ, R., TÉBAR, J. y ABAD, L. (2010): “Empleo y economía del conocimiento en la región metropolitana de Madrid: una revisión crítica", comunicación presentada en el XI Seminario Internacional RII celebrado en Mendoza (Argentina) entre los días 26 y 30 de octubre de 2010.

MÉNDEZ, R., TÉBAR, J. y ABAD, L. (2011): "Economía del conocimiento y calidad del empleo en la región metropolitana de Madrid: una perspectiva crítica"; en Scripta Nova. Revista Electrónica de Geografía y Ciencias Sociales, Vol XV, n ${ }^{\circ}$ 280. http://www.ub.edu/geocrit/sn/sn-380.htm 
MINISTERIO DE PESCA, AGRICULTURA Y ALIMENTACIÓN (1996): Comarcalización agraria de España. Madrid.

MOULAERT, F. y NUSSBAUMER, J. (2005): "The Social Region: Beyond the Territorial Dynamics of the Learning Economy"; en European Urban and Regional Studies, $\mathrm{n}^{\mathrm{o}} 12$ (1), pp. 45-64.

OBSERVATORIO EUROPEO LEADER (1997): Innovación y desarrollo rural. Serie Informes del Observatorio, $\mathrm{n}^{\circ}$ 2, Bruselas.

TORRES SALCIDO, G. y RAMOS CHÁVEZ, H. A. (2008): "Gobernanza y territorios. Notas para la implementación de políticas para el desarrollo"; en Revista Mexicana de Ciencias Políticas y Sociales, $\mathrm{n}^{\circ}$ 203, pp. 75-95.

TROITIÑO, M. A. ET AL.. (2006): "La experiencia del Patronato Madrileño de Áreas de Montaña como experiencia de cooperación y desarrollo sostenible en la Sierra Norte de Madrid"; en Romero, J. y Farinós, J. (eds.): Gobernanza territorial en España. Claroscuros de un proceso a partir del estudio de casos. Publicacions de la Universitat de València, Sevilla.

UTANDA MORENO, M. L. (1996): Geografía agraria de la Comarca de «Las Vegas». Ediciones Doce Calles. Aranjuez.

UTANDA MORENO, M. L. (2004): "La evolución histórica de la industria en la comarca de Las Vegas"; en VV.AA: Historia, clima y paisaje. Estudios geográficos en memoria del profesor Antonio López Gómez. Universitat de Valencia. 233249.

VANIER, M. (2000): "Qu'est-ce que le tiers espace ? Territorialités complexes et construction politique"; en Revue de géographie alpine, Vol. 88, n 1. pp. 105-113. 